\title{
Ring chromosome 14 syndrome
}

INSERM

\section{Source}

INSERM. (1999). Orphanet: an online rare disease and orphan drug data base. Ring chromosome 14 syndrome. ORPHA:1440

Ring chromosome 14 syndrome is characterized by intellectual deficit, retinal and skin pigmentation disorders, seizures, and dysmorphic features, including flat occiput, epicanthal folds, downward slanting eyes, flat nasal bridge, upturned nostrils, short neck, and large low set ears. 